PROCEEDINGS OF THE

AMERICAN MATHEMATICAL SOCIETY

Volume 138, Number 3, March 2010, Pages 987-995

S 0002-9939(09)10114-4

Article electronically published on October 21, 2009

\title{
ON THE POSITIVSTELLENSATZ IN WEYL'S ALGEBRA
}

\author{
JOULES NAHAS
}

(Communicated by Nigel J. Kalton)

\begin{abstract}
We use harmonic analysis tools on the Heisenberg group to prove decay and regularity properties for a certain class of partial differential equations. These properties allow us to relax a technical condition for a sum of squares decomposition for the Weyl algebra.
\end{abstract}

\section{INTRODUCTION}

Recently, functional analysis has been used to characterize when certain algebraic objects are positive. Positive polynomials on semialgebraic sets, free $*$-algberas, and classes of operators on a function space can be characterized by this method. These results, called Positivstellensätze, proscribe that positive elements can be written as a sum of square elements. For a review, as well as applications of Positivstellensätze, see 6 .

Let $W(d)$ denote the Weyl algebra in $d$ dimensions. This is the (complex) algebra with $2 d$ generators $p_{1}, \ldots p_{d}, q_{1}, \ldots, q_{d}$, identity element $\mathbf{1}$, and the relations

$$
\begin{aligned}
p_{k} q_{l}-q_{l} p_{k} & =-i \delta_{k l} \mathbf{1}, \\
p_{k} p_{l}-p_{l} p_{k} & =0, \\
q_{k} q_{l}-q_{l} q_{k} & =0 .
\end{aligned}
$$

This algebra is commonly written in terms $a_{k} \equiv 2^{-\frac{1}{2}}\left(q_{k}+i p_{k}\right)$ and $a_{-k} \equiv 2^{-\frac{1}{2}}\left(q_{k}-\right.$ $i p_{k}$ ). Assuming some integrability conditions on the generators (see [5]), the Stonevon Neumann theorem states that there is only one irreducible representation of $W(d)$. This is the infinite dimensional representation, called the Schrödinger representation, that acts on $f \in \mathcal{S}\left(\mathbb{R}^{d}\right)$, the Schwartz space, as follows:

$$
\begin{aligned}
& p_{k} f=-i \frac{\partial}{\partial x_{k}} f, \\
& q_{k} f=x_{k} f .
\end{aligned}
$$

Because of this, we identify elements of $W(d)$ with differential operators having polynomial coefficients. We use this identification to introduce a grading $W(d)=$ $\bigoplus_{n \in \mathbb{N}} A_{n}$, where $A_{n}$ consists of the elements of $k \in W(d)$ whose principal symbol $k\left(x_{1}, \ldots, x_{d}, \xi_{1}, \ldots, \xi_{d}\right)$ is a polynomial of degree $n$ in the variables $x_{1}, \ldots, x_{d}$ and $\xi_{1}, \ldots, \xi_{d}$.

Received by the editors October 10, 2008, and, in revised form, November 9, 2008, and July 8, 2009

2010 Mathematics Subject Classification. Primary 47L80.

(C)2009 American Mathematical Society Reverts to public domain 28 years from publication 
This representation also allows us to define the set $W(d)_{+} \subset W(d)$ of elements that are positive in the sense of operators on $L^{2}\left(\mathbb{R}^{d}\right)$ having domain $\mathcal{S}\left(\mathbb{R}^{d}\right)$. Let

$$
\Sigma W(d)^{2}=\left\{a \in W(d): a=\sum_{i=1}^{k} x_{i}^{*} x_{i} \text { with } x_{i} \in W(d)\right\} .
$$

Define $\mathbf{N}$ to be the subset of $W(d)$ consisting of all finite products of the form $\sum_{k=1}^{d}\left(q_{k}+i p_{k}\right)^{*}\left(q_{k}+i p_{k}\right)+(n+\alpha) \mathbf{1}$ for $n \in \mathbb{Z}$ and some $\alpha \in \mathbb{R}$. For convenience, we let $x$ and $\xi$ denote the vectors in $\mathbb{R}^{d},\left(x_{1}, \ldots, x_{d}\right)$ and $\left(\xi_{1}, \ldots, \xi_{d}\right)$ respectively. In [8], Schmüdgen proved the following Positivstellensatz for the Weyl algebra in the Schrödinger representation.

Theorem 1.1. Let $c$ be a hermitian element of the Weyl algebra $W(d)$ of even degree $2 m$ and let $c_{2 m}(x, \xi)$ be the principal symbol of the degree $2 m$ term of $c$. Assume that

(1) there exists $\epsilon>0$ such that $c-\epsilon * 1 \in W(d)_{+}$;

(2) $c_{2 m}(x, \xi)>0$ for all $(x, \xi) \in \mathbb{R}^{2 d},(x, \xi) \neq(0,0)$.

If $m$ is even, then there is an element $b \in N$ such that $b c b \in \Sigma W(d)^{2}$. If $m$ is odd, then there exists $b \in \boldsymbol{N}$ such that $\Sigma_{j=1}^{d} b a_{j} c a_{j}^{*} b \in \Sigma W(d)^{2}$.

The first condition is only needed in this theorem to assume the validity of the following statement: Let $y_{0}=\left(-\Delta+|x|^{2}+\alpha\right)^{-1}$. If for all nonzero $\phi \in \mathcal{S}\left(\mathbb{R}^{d}\right)$, $\left\langle y_{0}^{m} c y_{0}^{m} \phi, \phi\right\rangle>0$, then for all nonzero $\phi \in L^{2}\left(\mathbb{R}^{d}\right),\left\langle y_{0}^{m} c y_{0}^{m} \phi, \phi\right\rangle>0$. Our main result, Theorem 4.1, proves that for $\phi \in L^{2}\left(\mathbb{R}^{d}\right)$,

$$
\left\langle y_{0}^{m}{ }^{-} y_{0}^{m} \phi, \phi\right\rangle=0 \Longrightarrow \phi \in \mathcal{S}\left(\mathbb{R}^{d}\right) \text {. }
$$

This proves the following Positivstellensatz.

Theorem 1.2. Let $c$ be a hermitian element of the Weyl algebra $W(d)$ of even degree $2 m$ and let $c_{2 m}(x, \xi)$ be the principal symbol of the degree $2 m$ term of $c$. Assume that

(1) The element $c$ is strictly positive on $\mathcal{S}\left(\mathbb{R}^{d}\right)$.

(2) $c_{2 m}(x, \xi)>0$ for all $(x, \xi) \in \mathbb{R}^{2 d},(x, \xi) \neq(0,0)$.

If $m$ is even, then there is an element $b \in N$ such that $b c b \in \Sigma W(d)^{2}$. If $m$ is odd, then there exists $b \in N$ such that $\Sigma_{j=1}^{d} b a_{j} c a_{j}^{*} b \in \Sigma W(d)^{2}$.

Theorem 4.1 is proved by studying the solutions $u$ of partial differential equations of the form

$$
c u=f .
$$

We use techniques from the theory of pseudo-differential operators, which we introduce in section 2. These are used to derive regularity and decay properties in section 3, which in turn, prove statement (1.1) in section 4 .

\section{Harmonic analysis ON $\mathbf{H}$}

We recall some definitions and a theorem from harmonic analysis on the Heisenberg group, but refer the reader to [4] and [3] for a more complete treatment. The Heisenberg group of a vector space $X$ of dimension $n$ is the set $\mathbf{H}=X \times X^{*} \times \mathbb{T}$ under the group operation

$$
\left(x_{1}, y_{1}, z_{1}\right) *\left(x_{2}, y_{2}, z_{2}\right)=\left(x_{1}+x_{2}, y_{1}+y_{2}, z_{1} z_{2} e^{i y_{1}\left(x_{2}\right)}\right) .
$$


Let the group $\mathbf{H}$ act on functions on $X$ under the action $\rho$ as follows:

$$
\rho\left(x^{\prime}, \xi^{\prime}, z^{\prime}\right) f(x) \equiv z^{\prime} e^{i \xi^{\prime}\left(x-x^{\prime}\right)} f\left(x-x^{\prime}\right) .
$$

This $\rho$ can be used to map a distribution on $\mathbf{H}$ to a distribution on $X$ in the following manner. If $D \in \mathcal{S}^{*}(\mathbf{H})$, then

$$
D(\rho(f))=D\left(z^{\prime} e^{i \xi^{\prime}\left(x-x^{\prime}\right)} f\left(x-x^{\prime}\right)\right) .
$$

We call this new distribution $\rho(D)$. Let exp denote the exponential map from the corresponding Lie algebra $\mathfrak{h}$ to $\mathbf{H}$. We will restrict our concern to the set

$$
\mathcal{S}(H, e) \equiv\left\{f \in \mathcal{S}(H): f(x, \xi, z)=\frac{1}{z} f(x, \xi, 1)\right\}
$$

and the associated space of distributions. Let the map lg: $X \cup X^{*} \rightarrow \mathfrak{h}$ be defined by the equation $\exp (\lg x)=(x, 0,1)$ if $x \in X$. For $y \in X^{*}$, we let $\lg y$ be the element that satisfies $\exp (\lg y)=(0, y, 1)$. Then $\mathfrak{h}$ can be identified with $\lg X \oplus \lg X^{*} \oplus \mathbb{R}$. Consider the set $\left\{\left(x, \xi, e^{\frac{i}{2} \xi(x)}\right)\right\} \subset \mathbf{H}$. This is simply the image of $W \equiv \lg X \oplus \lg X^{*}$ under the exponential map. Let $f \in \mathcal{S}(W)$. We define a symplectic form on $W$ by

$$
\langle\lg x, \lg \xi\rangle_{W} \equiv \xi\left(x^{\prime}\right)-\xi^{\prime}(x) .
$$

Then define a natural Fourier transform on $W$ as follows:

$$
\hat{f}\left(w^{\prime}\right) \equiv 2^{-n} \int_{W} f(w) e^{\frac{i}{2}\left\langle w, w^{\prime}\right\rangle_{W}} d w .
$$

There is a canonical map $s$ between $\mathcal{S}(H, e)$ and $\mathcal{S}(W)$ defined by

$$
s(f)(w) \equiv f(\exp w) .
$$

We use this map to pull back distributions on $W$, which can be Fourier transformed, into $\mathcal{S}^{*}(H, e)$, where the distributions can act on $\mathcal{S}(X)$. Namely, if $D \in \mathcal{S}^{*}(W)$, we call $\hat{D}$ the isotropic symbol of $\rho\left(s^{-1}(D)\right)$.

The isotropic symbol of a differential operator is closely related to the principal symbol of a differential operator. Let $X=\mathbb{R}^{d}$. It is easy to show that if $D$ is a differential operator on $X$ with polynomial coefficients, then the isotropic symbol of $D$ will be a polynomial. Moreover, the highest degree terms of the principal and isotropic symbol in the sense of $\bigoplus_{n \in \mathbb{N}} A_{n}$ will be the same.

We will abuse notation slightly and let $\hat{E}$ denote the isotropic symbol of a differential operator $E$ on $X$. The isotropic symbol is convenient to work with, because of the following theorem, found in [4]:

Theorem 2.1. Let $\exp \left(\frac{-1}{2 \pi} \Delta\right)$ denote the operator

$$
\begin{aligned}
& 1-\frac{1}{2 \pi} \sum_{i=1}^{d}\left(\frac{\partial^{2}}{\partial x_{i}^{2}}+\frac{\partial^{2}}{\partial \xi_{i}^{2}}\right) \\
& +\frac{1}{2 !}\left(\frac{1}{2 \pi}\right)^{2} \sum_{i, j=1}^{d}\left(\frac{\partial^{4}}{\partial x_{i}^{2} \partial x_{j}^{2}}+2 \frac{\partial^{4}}{\partial x_{i}^{2} \partial \xi_{j}^{2}}+\frac{\partial^{4}}{\partial \xi_{i}^{2} \partial \xi_{j}^{2}}\right)-\ldots
\end{aligned}
$$

If $D \in \mathcal{S}^{*}(W)$ has compact support, a sufficient condition for $\rho\left(s^{-1}(D)\right)$ to be positive is for

$$
\exp \left(\frac{-1}{2 \pi} \Delta\right)(\hat{D})
$$

to be positive. 
For $p \in \mathbb{C}[x, \xi]$, the operator $\exp \left(\frac{-1}{2 \pi} \Delta\right)$ has the standard inverse:

$$
\exp \left(\frac{-1}{2 \pi} \Delta\right) \exp \left(\frac{1}{2 \pi} \Delta\right) p=p
$$

Let $E \in W(d)$ be such that the principal symbol of the highest degree terms obeys condition 2 of Theorem 1.2. Theorem 2.1 says that $E$ is positive, except for lower degree terms. We will see that lower degree terms can be added to make $E$ positive. Furthermore, this positive operator can be perturbed and still remain positive. We do this with the following lemmas.

Lemma 2.1. Suppose $p(x, \xi)$ is a homogenous polynomial of degree $2 m$ that satisfies

$$
p(x, \xi) \geq 0,
$$

with equality only when $x=\xi=0$. Then there exists a $\beta \in \mathbb{R}_{+}$so that the polynomial

$$
p(x, \xi)-\beta \sum_{i=1}^{d}\left(x_{i}^{2 m}+\xi_{i}^{2 m}\right)
$$

satisfies condition 2.2 .

Proof. Let $v$ be the minimum of $p(x)$ on the manifold

$$
M \equiv\left\{(x, \xi) \in \mathbb{R}^{2 d}: x_{1}^{2 m}+\xi_{1}^{2 m} \ldots+x_{d}^{2 m}+\xi_{d}^{2 m}=1\right\},
$$

and let $\beta \equiv \frac{v}{2}$. Since $M$ is compact, $v$ is finite. It will suffice to show that the minimum of

$$
q(x, \xi) \equiv p(x, \xi)-\beta \sum_{i=1}^{d}\left(x_{i}^{2 m}+\xi_{i}^{2 m}\right)
$$

on $M$ is greater than 0 . This is because $p$ and $q$ are homogeneous polynomials, and for any nonzero $y \in \mathbb{R}^{2 d}$, there is a $y_{0} \in M$ and an $r \in \mathbb{R} \backslash\{0\}$ so that $r y_{0}=y$. This implies that

$$
q(y)=r^{2 m} q\left(y_{0}\right) .
$$

Therefore $q$ is determined by its values on $M$.

Since 0 is not in $M, v>0$. Then the minimum of $q$ on $M$ is $\frac{v}{2}$. Therefore $q$ is never 0 on $M$, and $q(x, \xi)=0$ only when $x=\xi=0$.

Lemma 2.2. Suppose $T \in W(d)$ has degree $2 m$ and that the principal symbol of the highest order term of $T$ satisfies condition 2 of Theorem [1.2. Then there is a $K \in W(d)$, with degree at most $2 m-1$, and $\beta \in \mathbb{R}_{+}$so that the operator

$$
\Omega=T-K-\beta \sum_{i=1}^{d}\left(x_{l}^{2 m}+\left(i \frac{\partial}{\partial x_{l}}\right)^{2 m}\right)
$$

is positive.

Proof. Let $p(x, \xi)$ be the principal symbol of the degree $2 m$ part of $T$. Because the highest degree terms of the principal and isotropic symbols are the same,

$$
\hat{T}(x, \xi)=p(x, \xi)+q(x, \xi),
$$

where $q$ is a polynomial of degree at most $2 m-1$. By Lemma 2.1, there is a $\beta \in \mathbb{R}_{+}$ such that

$$
p(x, \xi)-\beta \sum_{i=1}^{d}\left(x_{i}^{2 m}+\xi_{i}^{2 m}\right)
$$


satisfies (2.2). Let $r(x, \xi)$ be the polynomial

$$
\begin{aligned}
r(x, \xi) & =\left(\hat{T}-\beta \sum_{i=1}^{d}\left(x_{i}^{2 m}+\xi_{i}^{2 m}\right)\right) \\
& -\exp \left(\frac{1}{2 \pi} \Delta\right)\left(p(x, \xi)-\beta \sum_{i=1}^{d}\left(x_{i}^{2 m}+\xi_{i}^{2 m}\right)+1\right) .
\end{aligned}
$$

By (2.1),$r(x, \xi)$ obeys the following equation:

$$
\begin{aligned}
\exp \left(\frac{-1}{2 \pi} \Delta\right) & \left(\hat{T}-\beta \sum_{i=1}^{d}\left(x_{i}^{2 m}+\xi_{i}^{2 m}\right)-r(x, \xi)\right) \\
& =p(x, \xi)-\beta \sum_{i=1}^{d}\left(x_{i}^{2 m}+\xi_{i}^{2 m}\right)+1 .
\end{aligned}
$$

Let $K$ be the operator with $\hat{K}=r(x, \xi)$. Then $\Omega$, defined by (2.4), is positive by (2.5) and Theorem 2.1.

\section{DECAY AND REGULARITY OF (1.2)}

We prove a lemma that will assist with the operator $y_{0}$ that occurs in (1.1). We note that 8 does not impose any conditions on the number $\alpha$ in $y_{0}$, but this means that $y_{0}$ will not always be defined. To avoid this problem, we require $\alpha>0$.

Let $H^{s}\left(\mathbb{R}^{d}\right)$ denote the Sobolev space

$$
H^{s}\left(\mathbb{R}^{d}\right) \equiv\left\{f \in L^{2}\left(\mathbb{R}^{d}\right) \mid\left\|(1-\Delta)^{\frac{s}{2}} f\right\|_{2}<\infty\right\} .
$$

Lemma 3.1. If $f \in L^{2}\left(\mathbb{R}^{d}\right)$ and $\alpha>0$, then there exists a solution $u(x)$ of

$$
-\Delta u+|x|^{2} u+\alpha u=f,
$$

which satisfies $u \in H^{1}\left(\mathbb{R}^{d}\right) \cup L^{2}\left(|x|^{2} d x\right)$.

Proof. This follows immediately from defining an inner product

$$
\langle u, v\rangle \equiv \int_{\mathbb{R}^{d}}\left((\bar{\nabla} u) *(\nabla v)+|x|^{2} \bar{u} v+\alpha \bar{u} v\right) d x
$$

and the Riesz representation theorem.

If the function $f$ in (3.1) is in the space $H^{k}\left(\mathbb{R}^{d}\right) \cup L^{2}\left(|x|^{2 k} d x\right)$, then we would expect that the solution $u$ of (3.1) is in $H^{k+1}\left(\mathbb{R}^{d}\right) \cup L^{2}\left(|x|^{2 k+2} d x\right)$. This can be proven by looking at the fundamental solution of (3.1). We prove it here by using a more general argument that is of interest in its own right.

This theorem will apply to elements $c$ in the Weyl algebra, but we will operate on $c$ with operators that are not in $W(d)$. We can use the grading $\bigoplus_{n \in \mathbb{N}} A_{n}$ on the resulting operators. We say the degree of the operators $S=\left(1+|x|^{2}\right)^{\frac{1}{4}}$ or $R=(1-\Delta)^{\frac{1}{4}}$ is $\frac{1}{2}$, and we define the degree of an operator which is some combination of $S, R$, and something in $W(d)$ in the obvious manner. 
Theorem 3.1. Suppose that $c$ is a degree $2 m$ element of the Weyl algebra, and that the top degree part of $c$ satisfies condition 2 of Theorem 1.2 , Let $f \in H^{\frac{n}{2}}\left(\mathbb{R}^{d}\right) \cup$ $L^{2}\left(|x|^{n} d x\right)$, for $n \in \mathbb{N}$. If $u \in H^{m}(\mathbb{R}) \cup L^{2}\left(|x|^{2 m} d x\right)$ solves

$$
c u=f,
$$

then $u \in H^{m+\frac{n}{2}}(\mathbb{R}) \cup L^{2}\left(|x|^{2 m+n} d x\right)$.

Proof. The proof is similar to that of Theorem 4.3.2 in [4]. The statement is obviously true for $n=0$. We will prove the case $n>0$ by induction. To this end, assume that the statement is true for some $n \in \mathbb{N}$. Suppose that $f \in H^{\frac{n+1}{2}}\left(\mathbb{R}^{d}\right) \cup$ $L^{2}\left(|x|^{n+1} d x\right)$. Let $L$ denote the lower order terms of $c$, and $T$ the highest order terms. Then $u$ satisfies

$$
T u=-L u+f .
$$

Let $q$ be the pseudo-differential operator $\left(1+|x|^{2}\right)^{\frac{n+1}{4}}+(1-\Delta)^{\frac{n+1}{4}}$.

We need to ensure all necessary functions are in the domain of $q$. First note that by elliptic regularity, $u \in C_{l o c}^{\infty}\left(\mathbb{R}^{d}\right)$. Let

$$
\psi(x)= \begin{cases}\left(\int_{(-1,1)^{d}} \exp \left(\frac{-1}{1-|y|^{2}}\right) d y\right)^{-1} \exp \left(\frac{-1}{1-|x|^{2}}\right) & \text { if } x \in(-1,1)^{d} \\ 0 & \text { otherwise }\end{cases}
$$

and let $\phi=\psi * \chi_{(-a, a)}$ for some open interval $(-a, a)$.

We apply $q(\phi \cdot)$ to (3.2) and commute it to the right:

$$
T(q \phi u)+[T, q \phi] u=-L(q \chi u)+[-L, q \phi] u+q(\phi f) .
$$

The commutator terms are easy to estimate, because

$$
\begin{aligned}
{\left[\left(1+|x|^{2}\right)^{\frac{n+1}{4}}, x_{k}\right] } & =0, \\
{\left[\left(1+|x|^{2}\right)^{\frac{n+1}{4}}, \frac{\partial}{\partial x_{k}}\right] } & =-\left(\frac{n+1}{2}\right) x_{k}\left(1+|x|^{2}\right)^{\frac{n-3}{4}},
\end{aligned}
$$

and the commutators for $(1-\Delta)^{\frac{n+1}{4}}$ are just the Fourier transform of the above commutators. Therefore all terms in (3.3), except for $T(q \phi u)$, have degree at most $2 m+\frac{n-1}{2}$.

By our assumptions on $c$ we can apply Lemma 2.2 to the operator $T$. From this, we have a $\beta \in \mathbb{R}_{+}$and $K \in W(d)$ of degree (at most) $2 m-1$ that satisfy (2.4). We add and subtract $K$ from $T$ in (3.3):

$$
(T-K)(q \phi u)+K(q \phi u)+[T, q \phi] u=-L(q \chi u)+[-L, q \phi] u+q(\phi f) .
$$

We multiply (3.4) by $q \phi \bar{u}$, then integrate. This yields:

$$
\begin{aligned}
& \int_{\mathbb{R}^{d}}((q \phi \bar{u})(T-K)(q \phi u)+(q \phi \bar{u}) K(q \phi u)+(q \phi \bar{u})[T, q \phi] u) d x \\
& =\int_{\mathbb{R}^{d}}(-(q \phi \bar{u}) L(q \phi u)+(q \phi \bar{u})[-L, q \phi] u) d x+(q \phi \bar{u})(q \phi f) .
\end{aligned}
$$

Except for $(q \phi \bar{u})(T-K)(q \phi u)$ and $(q \phi \bar{u})(q \phi f)$, all of the terms in the above equation will have at most degree $2 m+n$. These will be bounded, up to multiplication by a constant $C$, by

$$
\|u\|_{H^{m+\frac{n}{2}}}^{2}+\|u\|_{L^{2}\left(|x|^{2 m+n} d x\right)}^{2}+\|u\|_{2}^{2} .
$$


By the induction hypothesis, this is finite. Using the Cauchy-Schwarz inequality, the $(q \phi \bar{u})(q \phi f)$ term will be bounded by

$$
\left(\|u\|_{H^{\frac{n+1}{2}}}+\|u\|_{L^{2}\left(|x|^{n+1} d x\right)}+\|u\|_{2}\right)\left(\|f\|_{H^{\frac{n+1}{2}}}+\|f\|_{L^{2}\left(|x|^{n+1} d x\right)}+\|f\|_{2}\right) .
$$

Combining these bounds yields:

$$
\begin{aligned}
& \int_{\mathbb{R}^{d}}(q \phi \bar{u})(T-K)(q \phi u) d x \leq \\
& C\left(\|u\|_{H^{m}}^{2}+\|u\|_{L^{2}\left(|x|^{2 m} d x\right)}^{2}+\|u\|_{2}^{2}\right. \\
& +\left(\|u\|_{H^{\frac{n+1}{2}}}+\|u\|_{L^{2}\left(|x|^{n+1} d x\right)}+\|u\|_{2}\right)^{2} \\
& \left.+\left(\|f\|_{H^{\frac{n+1}{2}}}+\|f\|_{L^{2}\left(|x|^{n+1} d x\right)}+\|f\|_{2}\right)^{2}\right) .
\end{aligned}
$$

Since $2 m+n \geq n+1$ and $f \in H^{\frac{n+1}{2}}\left(\mathbb{R}^{d}\right) \cup L^{2}\left(|x|^{n+1} d x\right)$, it follows that (3.5) is finite.

We use $\beta$ to write (3.5) as

$$
\begin{aligned}
& \int_{\mathbb{R}^{d}}(q \phi \bar{u})\left(T-K-\beta \sum_{l=1}^{d}\left(x_{l}^{2 m}+\left(i \frac{\partial}{\partial x_{l}}\right)^{2 m}\right)\right)(q \phi u) d x \\
& +\int_{\mathbb{R}^{d}}(q \phi \bar{u}) \beta \sum_{l=1}^{d}\left(x_{l}^{2 m}+\left(i \frac{\partial}{\partial x_{l}}\right)^{2 m}\right)(q \phi u) d x<\infty .
\end{aligned}
$$

The operator $T-K-\beta \sum_{i=1}^{d}\left(x_{l}^{2 m}+\left(i \frac{\partial}{\partial x_{l}}\right)^{2 m}\right)$ is positive by Theorem 2.1 and the operator $\beta \sum_{i=1}^{d}\left(x_{l}^{2 m}+\left(i \frac{\partial}{\partial x_{l}}\right)^{2 m}\right)$ is positive by integration by parts. This implies that

$$
\int_{\mathbb{R}^{d}}(q \phi \bar{u}) \beta \sum_{l=1}^{d}\left(x_{l}^{2 m}\right)(q \phi u) d x<\infty
$$

and

$$
\int_{\mathbb{R}^{d}}(q \phi \bar{u}) \beta \sum_{l=1}^{d}\left(i \frac{\partial}{\partial x_{l}}\right)^{2 m}(q \phi u) d x<\infty .
$$

From (3.5), the bound on these terms will be independent of $a$. We take $a \rightarrow \infty$. This and (3.6) imply that $q u \in L^{2}\left(|x|^{2 m} d x\right)$. Inequality (3.7) implies that $q u \in H^{m}$. By the definition of $q$, it follows that

$$
u \in L^{2}\left(|x|^{2 m+n+1} d x\right) \cap H^{m+\frac{n+1}{2}} .
$$

This proves the induction step, which in turn proves the result.

Corollary 3.1. For $k \in \mathbb{Z}, k \geq 0$, let the function $f$ in (3.1) be in the space $H^{k}\left(\mathbb{R}^{d}\right) \cup L^{2}\left(|x|^{2 k} d x\right)$. Then the solution $u$ of (3.1) satisfies

$$
u \in H^{k+1}\left(\mathbb{R}^{d}\right) \cup L^{2}\left(|x|^{2 k+2} d x\right) .
$$

Proof. If $k=0$, the statement is true by Lemma 3.1. For $k>0$, the conclusion follows by applying Theorem 3.1 to the equation

$$
c u \equiv-\Delta u+|x|^{2} u+\alpha u=f .
$$




\section{Proof of (1.1)}

The spaces $H^{\infty}\left(\mathbb{R}^{d}\right)$ and $L^{2}\left(|x|^{\infty} d x\right)$ respectively denote $\bigcap_{s \in \mathbb{Z}^{+}} H^{s}\left(\mathbb{R}^{d}\right)$ and $\bigcap_{s \in \mathbb{Z}^{+}} L^{2}\left(|x|^{s} d x\right)$. It is easy to prove that

$$
H^{\infty}\left(\mathbb{R}^{d}\right) \cap L^{2}\left(|x|^{\infty} d x\right)=\mathcal{S}\left(\mathbb{R}^{d}\right) .
$$

We are ready to relax condition 1 in Theorem 1.2

Theorem 4.1. Let $c$ be a hermitian element of the Weyl algebra $W(d)$ of even degree $2 m$, and let $c_{2 m}(x, \xi)$ be the principal symbol of the degree $2 m$ term of $c$. Suppose that $c_{2 m}$ satisfies condition 2 of Theorem 1.2 . Then for $\phi \in L^{2}\left(\mathbb{R}^{d}\right)$,

$$
\left\langle y_{0}^{m}{ }^{-} y_{0}^{m} \phi, \phi\right\rangle=0 \Longrightarrow \phi \in \mathcal{S}\left(\mathbb{R}^{d}\right) .
$$

Proof. By Corollary 3.1, if $y_{0}^{m}$ has domain $L^{2}\left(\mathbb{R}^{d}\right)$, then the range is $H^{m}\left(\mathbb{R}^{d}\right) \cup$ $L^{2}\left(|x|^{2 m} d x\right)$. Therefore, the domain of $y_{0}^{m} c y_{0}^{m}$ is $L^{2}\left(\mathbb{R}^{d}\right)$. If $f \in L^{2}\left(\mathbb{R}^{d}\right)$ is such that

$$
\left\langle f, y_{0}^{m} c y_{0}^{m} f\right\rangle=\left\langle y_{0}^{m} f, c y_{0}^{m} f\right\rangle=0,
$$

then $g \equiv y_{0}^{m} f$ satisfies $\langle g, c g\rangle=0$. Since $c$ is a nonnegative hermitian operator on $L^{2}\left(|x|^{2 m} d x\right) \cap H^{m}\left(\mathbb{R}^{d}\right)$, there is a hermitian operator $\sqrt{c}$ with the same domain such that $\sqrt{c} \sqrt{c}=c$. Therefore $g$ satisfies $\langle\sqrt{c} g, \sqrt{c} g\rangle=\|\sqrt{c} g\|=0$. This implies $\sqrt{c} g=0$, so $c g=0$.

The function $g$ is a solution to the equation $c g=0$ in the weak sense, and $g \in H^{m}\left(\mathbb{R}^{d}\right) \cup L^{2}\left(|x|^{2 m} d x\right)$. By applying Theorem 3.1 to $c g=0$, it follows that $g \in H^{\infty}\left(\mathbb{R}^{d}\right) \cap L^{2}\left(|x|^{\infty} d x\right)$. Therefore,

$$
u=\left(-\Delta+|x|^{2}+\alpha\right)^{m} g \in \mathcal{S}\left(\mathbb{R}^{d}\right) .
$$

\section{ACKNOWLEDGMENT}

I would like to thank Mihai Putinar for introducing me to this subject and for many fruitful discussions.

\section{REFERENCES}

[1] R. Bochnak, M. Coste, M-F. Roy, Géométrie algébrique réelle, Springer-Verlag, Berlin, 1980.

[2] L.C. Evans, Partial Differential Equations, American Mathematical Society, Providence, RI, 2000. MR 1625845 (99e:35001)

[3] G. B. Folland, Harmonic Analysis in Phase Space, Princeton University Press, Princeton, NJ, 1989. MR983366 (92k:22017)

[4] R. Howe, Quantum Mechanics and Partial Differential Equations, J. Funct. Anal. 38 (1980), 188-254. MR587908 (83b:35166)

[5] T. Kato, On the Commutation Relation $A B-B A=c$, Arch. Rat. Mech. Anal. 10 (1962), 273275. MR0151867(27:1850)

[6] M. Putinar, J. W. Helton, Positive Polynomials in Scalar and Matrix Variables, the Spectral Theorem, and Optimization, in Operator Theory, Structured Matrices, and Dilations, Theta, Bucharest, June 1988, pp. 229-306. MR2389626

[7] R. Richtmeyer, Principles of Advanced Mathematical Physics, Vol. 1, Springer, New York, 1978. 
[8] K. Schmüdgen, A Strict Positivstellensatz for the Weyl Algebra, Math. Ann. 331 (2005), 779-794. MR2148796 (2007h:47127)

[9] K. Schmüdgen, A Strict Positivstellensatz for Enveloping Algebras, Math. Z. 254 (2006), 641-653. MR2244371 (2007m:22007)

Department of Mathematics, University of California Santa Barbara, South Hall 6607, SANTa BARBara, CAlifornia 93106

E-mail address: nahas@math.uesb.edu 